Analytical Methods for Discriminating Stardust in Aerogel Capture Media

S. Brennan, H. A. Ishii, J. P. Bradley, K. Luening, K. Ignatyev, P. Pianetta

September 8, 2007

2007 Denver X-ray Conference

Colorado Springs, CO, United States

July 30, 2007 through August 3, 2007 
This document was prepared as an account of work sponsored by an agency of the United States Government. Neither the United States Government nor the University of California nor any of their employees, makes any warranty, express or implied, or assumes any legal liability or responsibility for the accuracy, completeness, or usefulness of any information, apparatus, product, or process disclosed, or represents that its use would not infringe privately owned rights. Reference herein to any specific commercial product, process, or service by trade name, trademark, manufacturer, or otherwise, does not necessarily constitute or imply its endorsement, recommendation, or favoring by the United States Government or the University of California. The views and opinions of authors expressed herein do not necessarily state or reflect those of the United States Government or the University of California, and shall not be used for advertising or product endorsement purposes. 


\title{
ANALYTICAL METHODS FOR DISCRIMINATING STARDUST IN AEROGEL CAPTURE MEDIA
}

\author{
Sean Brennan ${ }^{1}$, Hope A. Ishii ${ }^{2}$, John P. Bradley ${ }^{2}$, Katharina Luening ${ }^{1}$, Konstantin \\ Ignatyev $^{1}$, and Piero Pianetta ${ }^{1}$ \\ ${ }^{1}$ Stanford Synchrotron Radiation Laboratory, Stanford Linear Accelerator Center, Menlo \\ Park CA 94025 \\ ${ }^{2}$ Institute for Geophysics and Planetary Physics, Lawrence Livermore National \\ Laboratory, Livermore CA 94550
}

\begin{abstract}
Comet 81P/Wild 2's serendipitous orbit change to the inner solar system in 1974 offered researchers a rare opportunity to sample cometary material from the Kuiper belt, a repository of material left over from solar system formation $\sim 4.6 \mathrm{Gyr}$ ago. NASA's Stardust mission intercepted the comet in January 2004 and returned with material collected from its tail in January 2006. The cometary material, consisting of particles ranging from 10 microns down to $<2 \mathrm{~nm}$, was collected in aerogel, a very low density $(\sim 3$ $\mathrm{mg} / \mathrm{cm}^{3}$ ) silica foam, to minimize the effects of deceleration from $6.1 \mathrm{~km} / \mathrm{s}$. The entire deceleration track is extracted from the aerogel block as a pyramidal shape known as a keystone which can be mapped using x-ray fluorescence prior to extraction of terminal or intermediate particles for other analyses. One goal of the track mapping is to determine the bulk composition of the cometary material returned. Unfortunately, although the aerogel is predominantly $\mathrm{SiO}_{2}$, there are sufficient quantities of trace elements similar to those expected in the cometary material to require sophisticated discrimination techniques in order to decide whether a fluorescence map pixel contains only aerogel or both aerogel and cometary material. We have developed a dual threshold analysis approach for better distinguishing cometary material from aerogel contaminants and have applied it to five Stardust impact tracks and terminal particles. Here, we present aspects of the dual threshold approach and demonstrate its impact on track composition for one track.
\end{abstract}

\section{INTRODUCTION}

Stardust, a NASA Discovery class mission, returned to Earth in January, 2006 with dust captured from the tail of Comet 81P/Wild 2 [1,2]. Microscopic dust particles were captured at a relative velocity of $6.1 \mathrm{~km} / \mathrm{s}$ in low-density silica aerogel tiles and adjacent aluminum foils [3]. These Stardust samples are unique in our collections of extraterrestrial materials: They are the first samples returned from a known parent body that originated in the Kuiper belt beyond the gas giants. Prior to 1974, Wild 2 orbited at heliocentric distances beyond Jupiter, but a gravitational encounter with that planet sent it into a short-period orbit in the inner solar system and allowed the Stardust spacecraft to intercept the comet's coma within the orbit of Mars. Since Comet Wild 2 has only been in its new orbit for 6 revolutions it is likely to be little-altered and a good representative 
of the material in the Kuiper belt. It is expected that most small bodies in the Kuiper belt have experienced little thermal or aqueous processing since formation and are therefore relatively unaltered since the solar system formed $\sim 4.6$ billion years ago [4].

The focus of the synchrotron work is the elemental composition of the Stardust particles using $\mathrm{x}$-ray fluorescence. In parallel with our studies are studies by other teams using a wide variety of instrumentation including x-ray micro-diffraction, electron microscopy, infra-red spectroscopy, organic abundance, and isotopic analysis. In addition to the efforts at the Stanford facility research teams at 5 other synchrotron facilities around the world were also involved in the preliminary examination team. The results of this examination were published in a series of papers in a special issue of Science $[2,5,6,7,8,9,10]$. Since presenting the results of our preliminary examination, we have attempted to refine our methods for the challenging problem of properly identifying which pixels in our fluorescence maps truly contain cometary material and which only contain aerogel. As will be discussed below, this is complicated by the presence in the aerogel of elements other than silicon and oxygen which can mimic the cometary material. This refined analysis has also been submitted for publication [11]. The focus of this work is to discuss in more detail the techniques used to distinguish cometary material from the surrounding background of aerogel plus contaminants. While specific to the issue of Wild 2 sample return analysis, the methods here are generally applicable to those situations where a fluorescence signal of interest is intermixed with known background signals.

\section{EXPERIMENTAL}

We studied five Stardust deceleration tracks during Stardust Preliminary Examination. All were extracted from the same aerogel tile, Cell 44, in the form of individual aerogel "keystones" [12], shown as micrographs in Fig. 1 at approximately similar scale. Each keystone contains a single deceleration track, a cavity in the silica aerogel formed by the hypervelocity capture of a piece of cometary material (impactor). Some impactors fell apart on impact, generating multiple sub-tracks from a single entry hole, and some remained relatively intact. For both types, measurable cometary material is present along the track. The track lengths vary from approximately 0.3 to $3.3 \mathrm{~mm}$ and from conical, "carrot" shapes to more bulbous shapes. Details of the tracks can be found in the supplemental online material of [5] and in [11].

Tracks were mapped using a hard x-ray scanning fluorescence microprobe on the wiggler Beam Line 6-2 at the Stanford Synchrotron Radiation Laboratory. The microprobe uses Kirkpatrick-Baez final focusing optics and adjustable virtual source slits for a beam size of $2 \times 2$ microns $^{2}$ with $10^{9}$ photons/second ( $\left.\mathrm{ph} / \mathrm{s}\right)$. For efficient mapping of the deceleration track, the focused spot size was increased to $6 \times 15$ microns $^{2}$ with $2 \times 10^{10}$ $\mathrm{ph} / \mathrm{s}$. $\mathrm{Si}(111)$ monochromator crystals selected $14 \mathrm{keV}$ incident $\mathrm{x}$-rays for easy access to the $\mathrm{K}$ absorption edges of elements from $\mathrm{Si}$ through $\mathrm{Br}$. The microprobe is equipped with a Leica optical microscope and aluminum-coated low-Z mirror for $x$-ray line-of-sight viewing and positioning of the sample in the x-ray beam (Fig. 2). 

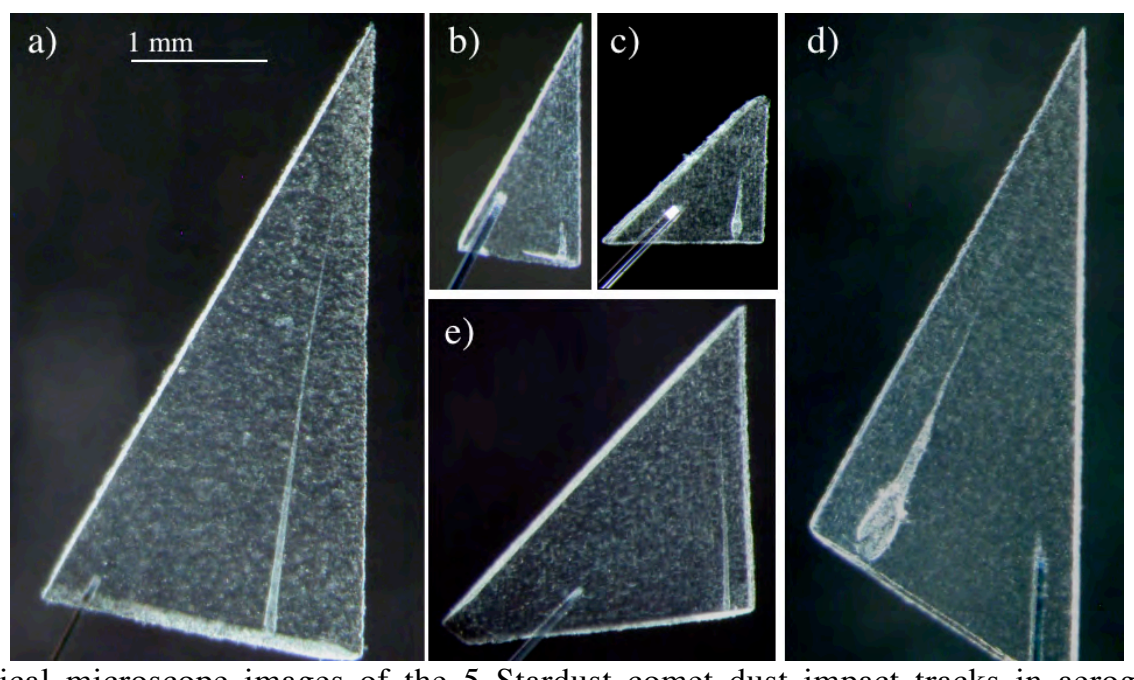

Fig. 1. Optical microscope images of the 5 Stardust comet dust impact tracks in aerogel keystones analyzed by micro-SXRF: a) Track 4, b) Track 5, c) Track 9, d) Track 10 and e) Track 12. Images are scaled to indicate relative sizes of tracks.

Keystones were translated in the beam in steps matched to the FWHM of the focused spot size and full fluorescence spectra were collected with dwell times of 30 seconds/pixel. Terminal particles and other particles along tracks were located primarily by high $\mathrm{Fe}$ fluorescence and scatter count rates. An ultra-clean $\mathrm{Si}(\mathrm{Li})$ detector with $\sim 150 \mathrm{eV}$ resolution $(\mathrm{Mn} \mathrm{K \alpha})$ was used to collect the fluorescent $x$-rays in a geometry perpendicular to the incident beam in the plane of the storage ring. An ion chamber before the sample and a PIN diode after were used to monitor the incident beam intensity. A helium shower covering the x-ray - sample interaction region reduces air absorption, ozone generation and Ar fluorescence. Recent improvements in that shower have essentially eliminated argon fluorescence from the spectra. (Fig. 2).

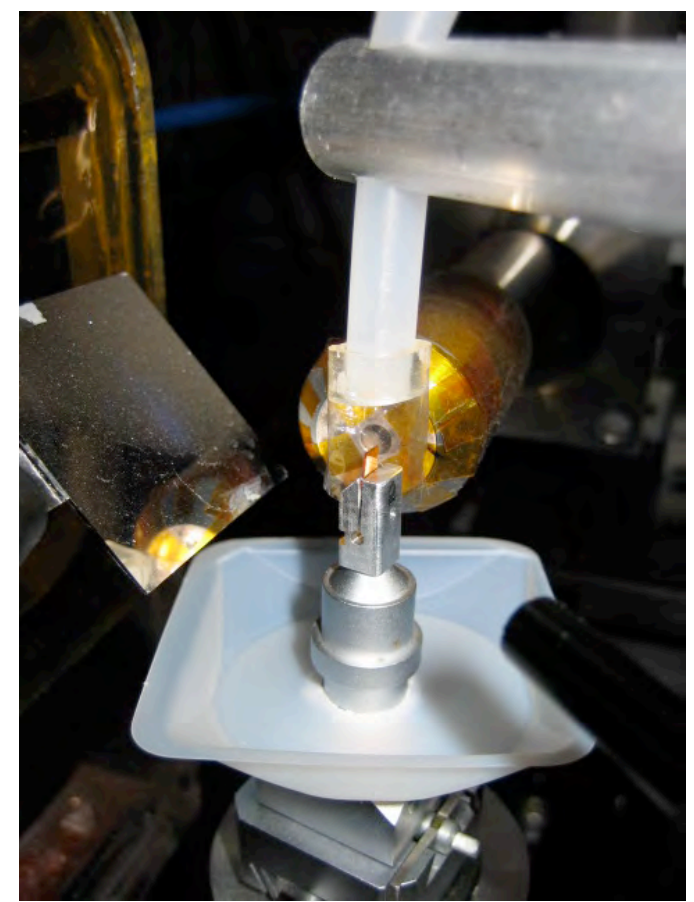

Fig. 2. Image of sample environment with polypropylene "shower curtain" surrounding sample. He flows over sample, eliminating Ar fluorescence as well as reducing elastic scatter.

Spectra were fit using PyMca, a program developed by Dr. Armando Sole at the European Synchrotron Radiation Facility (ESRF) and available for public use [13]. Whole track masses were derived from the difference between the sum of the on-track spectra containing cometary material and the normalized aerogel matrix background spectrum. The details of how this separation is achieved are discussed below. Two reference standards were employed in quantifying element masses: a thin $(200 \mathrm{~nm}) \mathrm{Fe}$ 
film acted as an absolute reference standard, and a USGS basaltic glass microprobe standard NKT-1G thin section $(200 \mathrm{~nm})$ was used to determine the energy-dependent correction for elements below Fe [14]. The NKT-1G basaltic glass standard contains $\sim 9.5 \mathrm{wt} \% \mathrm{Fe}$, so is especially useful for measuring self-absorption of low-Z fluorescence $\mathrm{X}$-rays (for example, sulfur) and corrects for detector response. What this standard does not address is the variation in self-absorption due to cometary particle size. Without specific knowledge of the cometary particle, the self-absorption can only be approximated. Thus, self-absorption is small for elements above $\mathrm{Ca}$, but considerably more uncertain for lower- $Z$ elements. The error in Fe mass is dominated by uncertainty in the thickness of the Fe standard and is at most a few percent.

\section{ANALYSIS}

One of the simplest and most powerful techniques is to use a region of interest (ROI) technique to identify where certain elements are present. One such map is presented in Fig. 3, showing the concentration of Fe along the deceleration track, with an optical image of the same track above it. The horizontal scale for the two images is the same, but the vertical scale of the fluorescence map is enlarged to more easily see the variability along the track. The warmth of the color in the map is related to the intensity of the Fe signal from that pixel; the data are scaled as the log of the intensity so that range of the map is not dominated by the very intense terminal particle. The distinction between the midnight and royal blue pixels on the periphery of the track are discussed below. There is strong evidence that $\mathrm{Fe}$ is deposited throughout the deceleration track, although in this case the majority of the Fe mass is at the terminal particle. There are other deceleration tracks where the majority of the Fe mass is distributed along the track, leaving only a small part of the total at the terminal particle.

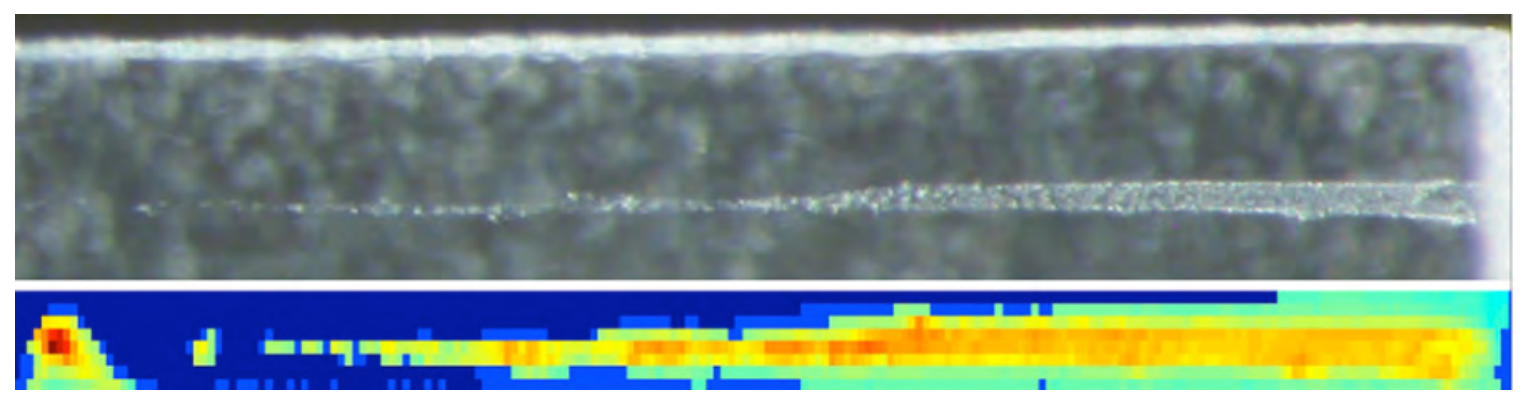

Fig. 3. Optical microscope image of track 12, enlarged to just see the track. Below it, the Fe fluorescence map of the same track, with an expanded vertical scale for clarity. The midnight blue is for aerogel only pixels, the royal blue is for the in-between pixels and warmer colors are where cometary material is found.

There are several challenges in calculating a total cometary mass in a deceleration track, most of them fundamentally related to the nature of the aerogel capture medium. Aerogel is a silica "foam". This fundamentally limits our ability to measure the presence of silicates in the cometary material. Where one would normally use the Si fluorescence signal as a reference for comparison to other kinds of cometary or meteoritic material, in this case, the Si fluorescence is dominated by the surrounding aerogel, especially as it has been shown that there is compressed aerogel surrounding the terminal particle. Typical aerogel keystone thicknesses are 100-500 microns, so silicates in the cometary material 
are completely masked by the surrounding aerogel. Analysis is further complicated by the presence of contamination within the aerogel, including $\mathrm{Fe}, \mathrm{Zn}, \mathrm{Cu}, \mathrm{Cr}$, Ti; many elements of interest in the comet are also potentially present in the aerogel. The surrounding aerogel is also effective in masking $\mathrm{Mg}$ and $\mathrm{Al}$ fluorescence that may be emanating from the cometary material, making those elements hard to quantify. Selfabsorption of low- $\mathrm{Z}$ elements $(<\mathrm{Ca}$, e.g.) increases the error bars on estimates of those masses.

We have tried several methods for identifying pixels that include cometary material. In the end, we have chosen the Fe fluorescence concentration in each pixel as the best metric. Another method, using the total scattered (elastic) signal from a pixel, was tested and found to be similar to but less reliable than using iron. We have chosen a dual threshold method: If the Fe concentration in a pixel was greater than some threshold, then it was considered to contain both cometary material and aerogel, if less than some lower threshold, than it contains only aerogel, and if the Fe concentration is between those two thresholds, it is discarded. The resulting spectra can be seen in Fig. 4, with the

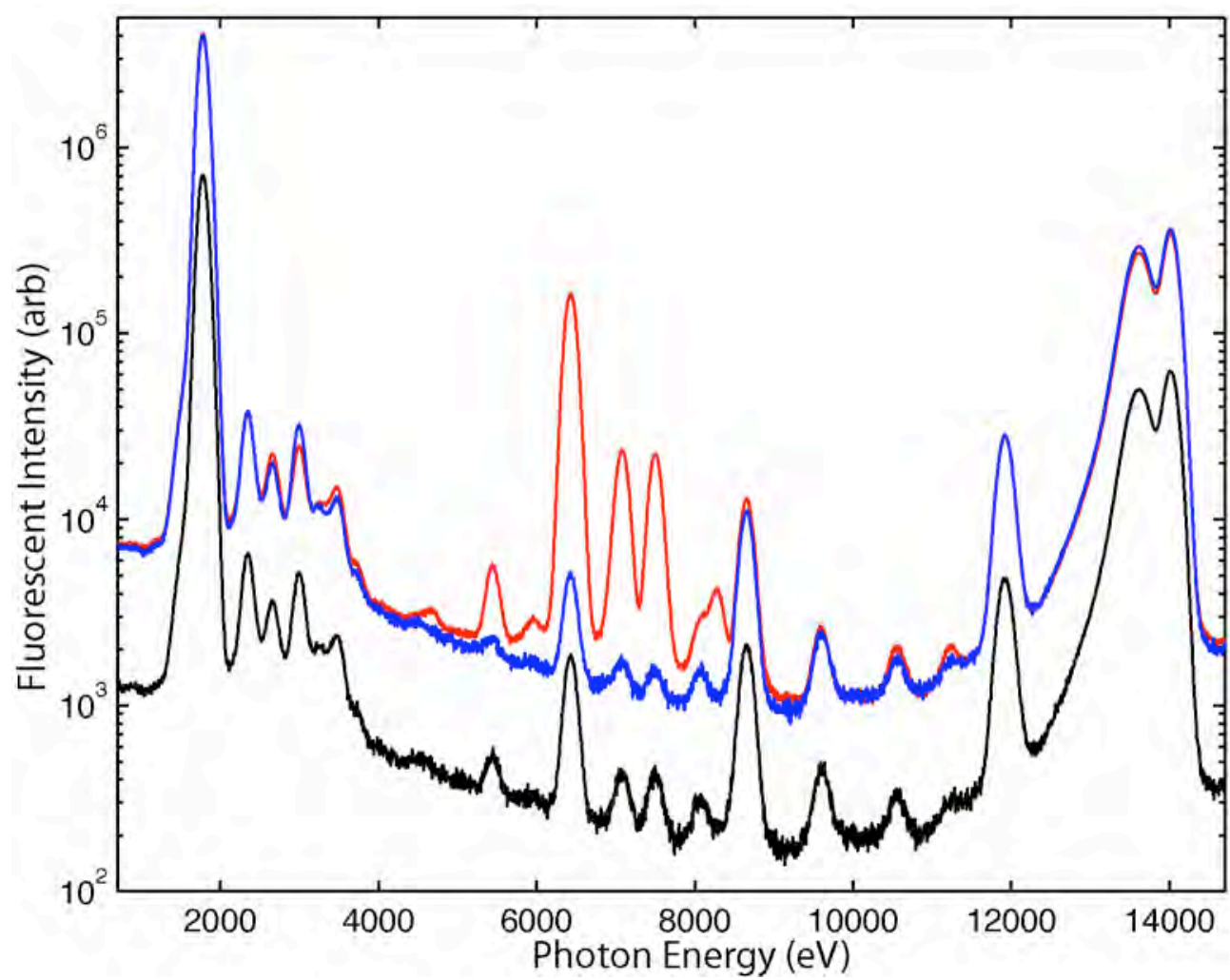

Fig. 4. Summed fluorescence spectra from the track map of track 12. The red curve is from those pixels which include both cometary and aerogel, the blue curve is from those that only contain aerogel, and the black curve is from those where we are uncertain. The blue curve has been scaled to the red curve, but the black curve is unscaled.

spectra plotted as the log of the fluorescence intensity vs. the photon energy in $\mathrm{eV}$. The red curve is the sum of those spectra thought to contain both cometary material and aerogel, the blue curve is the sum of those pixels which only contain aerogel, and the black curve the spectra for which we are undecided. Note that the blue curve has been 
normalized to have the same Si and scatter peak amplitude as the red curve, but the black curve has not been scaled. To give a sense of the scaling factors, the red curve represents almost 29000 seconds of collected data, the background just under 12000 seconds of data and the undecided spectrum is 5500 seconds of data. To see where the undecided pixels are on the map, compare the midnight-blue areas of Fig. 3 to the royal-blue areas. It is immediately clear that the undecided pixels are where one would expect to see them: on the periphery of the track, where the transition occurs from clearly cometary plus background to clearly background.

The reason it is important not to include these undecided spectra in either of the other two is because the cometary material is calculated by subtracting the results of analyzing the background spectrum from the results of analyzing the cometary spectrum. If cometary material is mistakenly included in the background spectrum, then one loses twice: cometary material missing from that spectrum is then subtracted from the remaining cometary material. If one is too free in including pixels without any cometary material

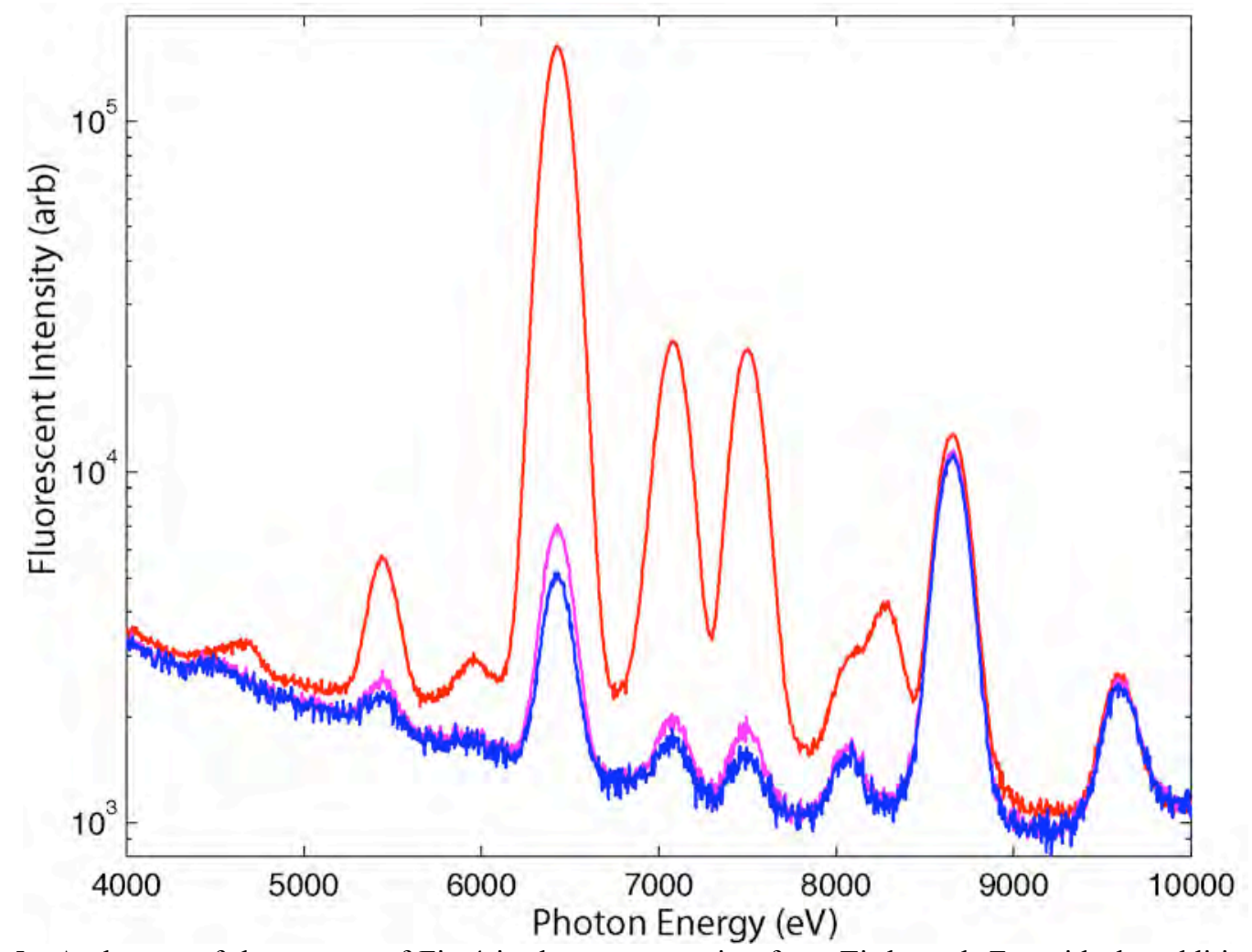

Fig. 5. A closeup of the spectra of Fig.4 in the energy region from Ti through $\mathrm{Zn}$, with the addition of a spectrum in magenta, which is the sum of the background and undecided spectra from Fig. 4, then scaled so that the elastic and Si peaks are the same as those of the cometary spectrum.

into that spectrum, then those elements with only trace concentrations will be effectively buried by the statistical noise of the additional pixels with only aerogel in them. The challenge is to select the thresholds so that one is sure that the background spectrum does not contain any cometary material, and that as little material is in the undecided spectrum as possible. 
To explore the effect of having this undecided bin for some of the pixels, consider Fig. 5, where instead of separating the undecided pixels, they are instead added to the background spectrum, effectively going to a single threshold method. One can see that, although the changes are small, there clearly are differences between the blue and magenta spectra. This is because the scatter/Fe ratio is different for the two, with more $\mathrm{Fe}, \mathrm{Ni}, \mathrm{Zn}$, etc present in the magenta (relative to the scatter peak) than in the true background spectrum. In practice, the lower threshold is determined by watching metrics such as the $\mathrm{Fe} / \mathrm{scatter}$ ratio as the threshold is changed, picking as the appropriate level that point where there is a significant change in that ratio. To more clearly see that the $\mathrm{Fe}$ and $\mathrm{Ni}$ (and especially $\mathrm{Zn}$ ) ratios to scatter peak are different between the background and undecided spectra, Fig. 6 shows the cometary, background and undecided spectra, now all scaled so that they have the same Si and scatter peak amplitudes.

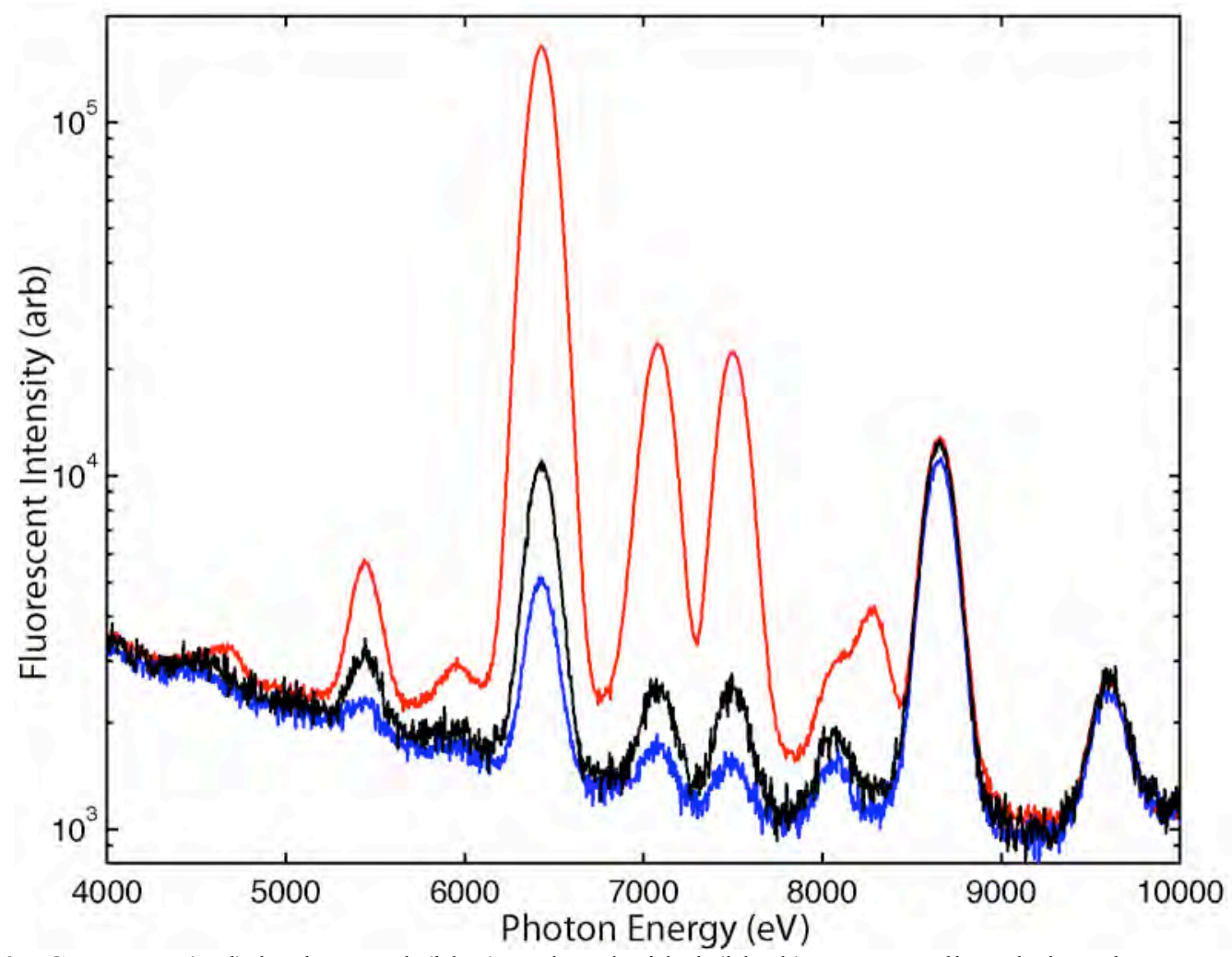

Fig. 6. Cometary (red) background (blue) and undecided (black) spectra, all scaled to the same Si and scatter intensities. Only the energy region from $\mathrm{Ti}$ to $\mathrm{Zn}$ is shown. The very different Fe-scatter and especially $\mathrm{Zn}$-scatter ratios are clear.

In this figure the very different ratios for Fe-scatter and especially Zn-scatter are clear. There is significantly more cometary material in these pixels than in the surrounding background pixels. Adding these pixels only has a small effect on the calculated $\mathrm{Fe}$ mass, but a very large effect on the lower-mass elements such as Zn. (Remember, these figures show the $\log$ of the fluorescence intensity). The results of analyzing these two approaches can be shown in Table 1, which compares the calculated masses of Track 12 using the two methods. 
Table 1. Element abundances by mass for Stardust impact Track 12 by single and dual threshold analysis.

\begin{tabular}{|c|ccc|}
\hline & $\begin{array}{c}\text { single threshold } \\
\text { mass (g) }\end{array}$ & $\begin{array}{c}\text { dual threshold } \\
\text { mass (g) }\end{array}$ & $\begin{array}{c}\text { fractional } \\
\text { change }\end{array}$ \\
\hline $\mathbf{C l}$ & $3.05 \mathrm{E}-11$ & $5.78 \mathrm{E}-11$ & 1.89 \\
$\mathbf{K}$ & $6.19 \mathrm{E}-12$ & $1.27 \mathrm{E}-11$ & 2.04 \\
$\mathbf{C a}$ & $2.07 \mathrm{E}-13$ & & \\
$\mathbf{C r}$ & $5.92 \mathrm{E}-12$ & $8.51 \mathrm{E}-12$ & 1.44 \\
$\mathbf{M n}$ & $4.37 \mathrm{E}-14$ & $6.17 \mathrm{E}-14$ & 1.41 \\
$\mathbf{F e}$ & $2.89 \mathrm{E}-10$ & $2.98 \mathrm{E}-10$ & 1.03 \\
$\mathbf{N i}$ & $2.48 \mathrm{E}-11$ & $2.58 \mathrm{E}-11$ & 1.04 \\
$\mathbf{C u}$ & $1.52 \mathrm{E}-13$ & $3.10 \mathrm{E}-13$ & 2.04 \\
$\mathbf{Z n}$ & $2.15 \mathrm{E}-13$ & $8.83 \mathrm{E}-13$ & 4.11 \\
$\mathbf{G a}$ & $2.12 \mathrm{E}-14$ & & \\
$\mathbf{A s}$ & $1.39 \mathrm{E}-13$ & $2.48 \mathrm{E}-13$ & 1.78 \\
$\mathbf{S e}$ & $2.09 \mathrm{E}-13$ & $2.05 \mathrm{E}-13$ & 0.98 \\
\hline total & $3.57 \mathrm{E}-10$ & $4.04 \mathrm{E}-10$ & 1.13 \\
\hline
\end{tabular}

* Measured masses above minimum detection limits are reported here.

While the total mass only changes marginally, the changes in some elements (notably $\mathrm{Zn}$, has been significant $(>4)$. The total mass is dominated by the $3 \%$ increase in the Fe mass, a small but significant change. One curious outcome of this analysis is that the sulfur signal does not rise above that measured in the background in either single or dual threshold analyses. Since collecting these data we have become suspicious that volatile elements may have a larger distribution perpendicular to the track than would be expected from looking at the Fe distribution. This conjecture will be tested in the near future.

\section{CONCLUSIONS}

The analysis of Stardust deceleration tracks has been an exciting project for our team and has raised a set of questions which are potentially common to a variety of fluorescence mapping projects. If there is an element of interest distributed in a matrix which includes low levels of contamination of that same element, how does one successfully isolate the material of interest? We have presented one such solution here, where one accepts that a limited number of pixels are "too close to call" and the consequences of guessing wrong can be significant, especially for trace elements which have not been used to make the segregation between signal and background. One can create guidelines of how one chooses to set the two thresholds, but we have yet to identify a rigorous method for their selection.

\section{ACKNOWLEDGEMENTS}

We gratefully acknowledge NASA and the Stardust Preliminary Examination Teams for the opportunity to study such exciting and unique samples. We also thank the Bulk Chemistry Subteam for useful interactions and discussions, and we acknowledge the enormous efforts of all involved in Curation to carry out rapid sample preparation and distribution. Portions of this work were carried out at the Stanford Synchrotron Radiation 
Laboratory, a national user facility operated by Stanford University on behalf of the U.S. Department of Energy, Office of Basic Energy Sciences. This work was performed in part under the auspices of the U.S. Department of Energy, National Nuclear Security Administration by the University of California, Lawrence Livermore National Laboratory, contract No. W-7405-Eng-48. This work was supported by NASA Grants NNH06AD67I (Stardust Participating Scientist) and NNH04AB49I (SRLIDAP).

\section{REFERENCES}

[1] Brownlee D.E., Tsou P., Anderson J.D., Hanner MS., Newburn R. L., Sekanina Z., Clark B.C., Hörz F., Zolensky M.E., Kissel J., McDonnell J.A.M., Sandford S.A. and Tuzzolino A.J. Journal of Geophysical Research, 2003, 108, SRD 1-1 - 1-15.

[2] Brownlee D.E. et al. (183 coauthors) Science 2006, 314, 1711-1716.

[3] Tsou, P., Brownlee D.E., Sandford S.A., Hörz F. and Zolensky M.E. Journal of Geophysical Research 2003 108, SRD 3-1 - 21.

[4] Irvine W.M. and Lunine J.I. In Comets II, Festou M.C., Keller H.U. and Weaver H.A, eds. University of Arizona Press: Tucson, 2004, 25-31.

[5] Flynn G.J. et al. (80 coauthors) Science 2006 314, 1731-1735.

[6] Hörz F. et al. (44 coauthors) Science 2006, 314, 1716-1719.

[7] Keller L.P. et al. (33 authors) Science 2006, 314, 1728-1731.

[8] McKeegan K.D. et al. (47 coauthors) Science 2006, 314, 1724-1728.

[9] Sandford S.A. et al. (55 coauthors) Science 2006, 314, 1720-1724.

[10] Zolensky M.E. et al. (75 coauthors) Science 2006, 314, 1735-1739.

[11] Ishii, H.A, Brennan, S, Bradley, J, Luening, K, Ignatyev, K, Pianetta, P. Meteoritics and Planetary Science $\mathbf{2 0 0 7}$ (in press).

[12] Westphal A. J., Snead C., Butterworth A., Graham G. A., Bradley J. P., Bajt S., Grant P. G., Bench G., Brennan S. and Pianetta P. Meteoritics \& Planetary Science 2004 39, 1375-1386.

[13] Solé V.A., Papillon E., Cotte M., Walter Ph. and Susini J. Spectrochimica Acta B, 2007 62, 63-68. The code is available online at http://www.esrf.fr/computing/bliss/downloads/index.html.

[14] This reference standard is available from the USGS:

http://minerals.cr.usgs.gov/geo_chem_stand/microanalytical_RM.html 